\title{
Mathematical Analysis of Dynamic Channel Selection in Indoor Mobile Wireless Communication Systems
}

\author{
Jan B. Punt, Dirk Sparreboom, Frank Brouwer, and Ramjee Prasad, Senior Member, IEEE
}

\begin{abstract}
Dynamic channel selection (DCS) is an algorithm for flexible resource sharing in mobile radio systems. The digital enhanced cordless telecommunications (DECT) standard implements a version of DCS based on time as well as frequency multiplexing. In this paper, mathematical models are developed to evaluate the probabilities of channel availability, desired carrier power, and the carrier-to-interference ratio (CIR) for a constant traffic load. These results can be used to compute the call setup blocking probability. The models reported in this study are based on a decentralized DCS according to the DECT standard specifications. The results show that blocking due to availability of a channel (resource blocking), not interference blocking, generally is the most important factor in overall call blocking. Furthermore, it is seen that the resource blocking is sensitive to the implementation of the portable sets. Also, with homogeneous static traffic, this type of DCS can be as good as fixed channel allocation (FCA) with respect to resource blocking.
\end{abstract}

Index Terms - Cordless telephone systems, DECT, dynamic channel allocation, isolated control, resource management.

\section{INTRODUCTION}

W IRELESS communication systems widely incorporate frequency reuse to achieve a high-spectrum efficiency. The reuse algorithm should be chosen to satisfy the specific needs of a system, e.g., capacity or flexibility. Dynamic channel allocation (DCA) is an efficient reuse algorithm that can deal with traffic fluctuations. The main feature of DCA is that the channels are reused according to some function of the situation. Fixed channel allocation (FCA), on the contrary, allocates the channels to specific areas at system setup. The European Telecommunications Standards Institute (ETSI) has devised a standard called digital enhanced cordless telecommunications (DECT). This DECT standard is concerned mainly with indoor communication. The standard has some promising features over systems using analog techniques and FCA: DECT offers high-speech quality combined with a flexible system setup. In DECT, the mobiles select the

Manuscript received September 21, 1994; revised January 11, 1996.

J. B. Punt, D. Sparreboom, and R. Prasad are with the Telecommunications and Traffic-Control Systems Group, Department of Electrical Engineering and Department of Information Technology and Systems, Delft University of Technology, Delft, The Netherlands (e-mail: d.sparreboom@et.tudelft.nl).

F. Brouwer is with Ericsson Business Mobile Networks, B.V., The Netherlands, and the Telecommunications and Traffic-Control Systems Group, Department of Electrical Engineering, Delft University of Technology, Delft, The Netherlands.

Publisher Item Identifier S 0018-9545(98)08143-2. channels that will be used. This type of DCA is therefore called dynamic channel selection (DCS).

A mathematical analysis would enable us to gain significant insight into the mechanisms that influence the performance of DCS systems. The DCS as described in DECT is a decentralized algorithm. The mobile stations select a base station and a channel depending on the total signal power received from the nearby base stations, the availability of the channels, and the cochannel interference.

To date any analysis on DCS has mainly been done on the basis of simulations [1]-[6]. This paper extends the mathematical investigation from [7] and [8]. The models discussed in this paper give mathematical results for the performance of a DCS system at the moment of call setup. The analysis will be mainly statistical because the discussed DCS algorithm operates decentrally, which makes it hard to model system states. The models start bottom up: first the individual procedures are looked into, whereafter statements are made about the system as a whole. The system setup we investigate is described in Section II. In Section III, the models for channel availability treat three different ways to find out which channels are available. In Section IV, we show models for the power level of the desired signal. They calculate the strongest power from two and three available base stations. The model for interference power in Section V uses a geographical area so defined that each channel can be assumed to be occupied by one user. Then, the received signal power (rsp) of each channel is known, and we can select the best of the available channels. The area may be extended by a separate outer area to accommodate a second user on each channel, which will improve accuracy. How the carrier-to-interference ratio (CIR) is calculated from carrier power and interference power is discussed in Section VI. The present investigations are a first step toward the calculation of the call-blocking probability.

\section{SySTEM CHARACTERISTICS}

The system discussed here is based on the DECT standard [9]-[11]. DECT works with channels that are created by using time-division multiple access (TDMA) as well as frequencydivision multiple access (FDMA). A constellation of 120 channels is created by multiplexing 12 time slots onto ten separate carriers. The duration of a frame of 12 time slots is 10 ms. Each group of 16 frames is called a multiframe. A constellation of 120 channels is shown in Fig. 1. 


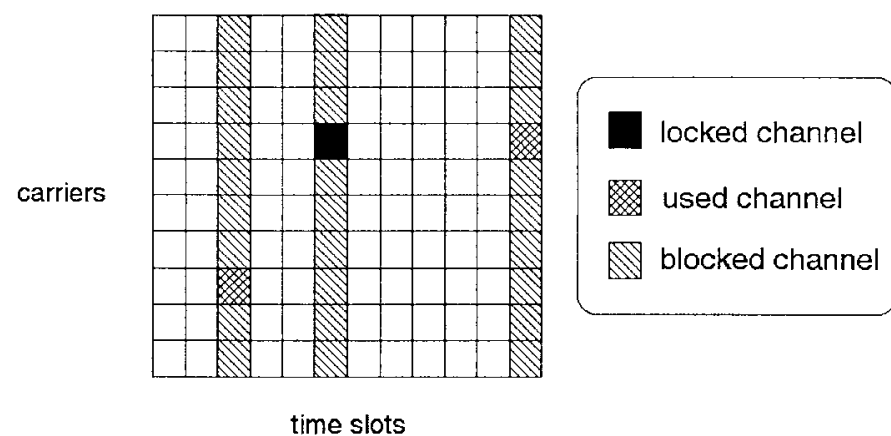

Fig. 1. Constellation of time- and frequency-multiplexed channels. Only downlink channels are displayed.

In the DECT standard, the initiative lies with the portables [portable parts (PP's)]. They find out which channel is the best one, and set up a call on the selected channel. The constellation in Fig. 1 represents the situation at a radio fixed part [(RFP) a base station]. Three channels are occupied. A PP that wants to set up a call is listening to one busy channel of the RFP. The RFP sends out identification and paging information on each busy channel. The channel that is used for this purpose by the PP is called the locked channel. Practical RFP's only have a single transceiver, which is fast enough to switch between different frequencies in between time slots. Because of this single transceiver, an RFP cannot be active on two frequencies in the same time slot. Thus, an RFP can handle a maximum of twelve calls, one in every time slot.

Within DECT, the quality of a communication link is expressed in the CIR. A value above $21 \mathrm{~dB}$ is considered acceptable. $11 \mathrm{~dB}$ of these 21 are a margin to account for $99 \%$ of the multipath effects. To keep the model tractable, the following list of system characteristics will be used throughout the paper.

- The average traffic per RFP is 5 Erlangs.

- An RFP can serve a maximum of 12 calls.

- We will assume that RFP's are always busy on at least one channel. In this way, the PP's are always provided with a channel to lock onto to obtain system and paging information. [For a conventional system with 12 lines (Erlangs 12 system) and a load of 5 Erlangs, this has a probability of $99.3 \%$.]

- Frames of different RFP's are assumed to be synchronized. Overlapping time slots would complicate the interference calculations. In practice, RFP's that are part of one DECT system will be synchronized.

- We use a model for the rsp that is valid only for distances larger than $1 \mathrm{~m}$ from the transmitter. To simplify the analysis, we disregard the area within $1 \mathrm{~m}$ of transmitters.

- The shadow fading is assumed to be uncorrelated in space and time.

- The shadow fading spread is defined as $1.5 \mathrm{~dB}$ at $1 \mathrm{~m}$ from the transmitter. It increases linearly to $8 \mathrm{~dB}$ at $10 \mathrm{~m}$. The value $1.5 \mathrm{~dB}$ was used for numerical reasons. From $10 \mathrm{~m}$ onwards, the spread is taken constant at $8 \mathrm{~dB}$.

- Noise power is taken to be $-104 \mathrm{dBm}$. This follows from a bandwidth of $1 \mathrm{MHz}$ and a noise figure of $10 \mathrm{~dB}$ at the receivers. It is not used unless indicated otherwise.
The constant noise power follows from the duration of the time slots: approximately $400 \mu$ s is long enough to average over enough periods to consider the noise power constant.

- The transmitted power used in the calculations is the maximum allowed by the DECT standard [10]: $250 \mathrm{~mW}$ (=24 dBm).

In this paper, results are only shown for distances between RFP's of $20 \mathrm{~m}$. With larger RFP distances the system becomes noise limited rather than interference limited.

\section{Channel Availability}

Whether a PP can use a channel at call setup is important. First, the PP looks for the strongest RFP. Then, at least one channel must be available (not already in use) at that RFP, i.e., the RFP must be free during at least one time slot. Last, at least one of the available channels must offer at least minimum performance, which means that the CIR should exceed 21 dB. When no channel can be used at this RFP (either no channels available or no channels offering enough quality), a PP may always try another RFP. The probability that no channel is available at the RFP selected by the PP is called the resource blocking probability. This is an important parameter to calculate call blocking. The probability that a nonzero number of channels is available is used in the calculations for interference power. In this section, we derive the probabilities of the number of available channels at an isolated

\section{A. Channel Scanning}

A PP needs to listen to an RFP to obtain system and paging information. A PP picks one of the active channels of an RFP and locks onto that. Next, to be able to select a channel for communication, the PP must have up-to-date information about the situation of the channels: whether they are in use and how much power it receives on them. For this purpose, PP's can scan channels. Based on the measured power level, the PP selects the best of the available channels it has scanned.

Today, commercially attractive PP's do not scan all possible channels for power level information. The scanning of all the channels would be energy consuming and complicated. We show that it might be advantageous, however, in terms of resource blocking.

We consider three methods of channel scanning. In the first one, channels are only scanned during the frame in which the PP listens to the locked channel. This need not be every frame. Each PP has only one transceiver, which is not fast enough to switch frequencies between two time slots. Therefore, only one carrier frequency can be scanned in a frame. The PP must also switch between the scanning carrier and the carrier of channel it is locked on (see Fig. 2). The transceiver switching takes too much time for the channels directly before and after the locked channel to be scanned. This is also called "neighboring channel blindness." The "neighboring" channels will not be used by the PP, since it does not know the quality of those channels. In the second method, we consider that within the neighboring time slots two channels can be scanned: the ones that are on the same carrier as the locked channel. When the 


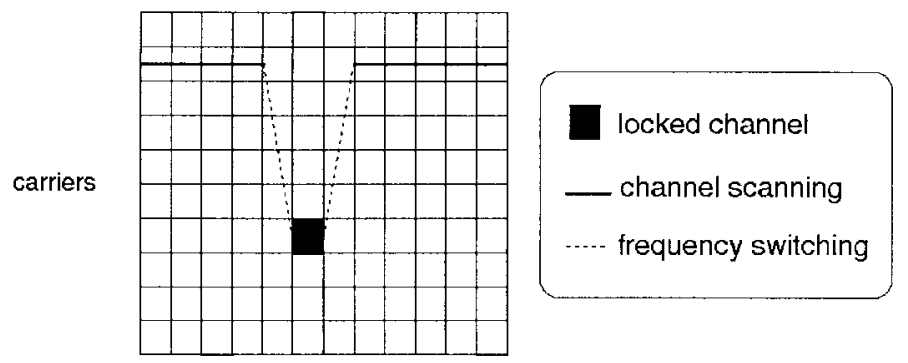

time slots

Fig. 2. The PP is scanning channels in the same frame in which it listens to the channel it is locked to. In between the scanning and listening, it has to switch frequencies.

mobile scans the channels on the carrier it is locked to, it does not switch frequency. In that case, two channels in the neighboring time slots can be scanned. This method does not increase the hardware complexity of a mobile. Third, a choice could be made to scan during other frames. In those frames, the PP need not listen to the locked channel. It is able to scan all the channels. The effect of the three different methods on the channel availability is investigated in the next sections. The results will be discussed at the end of Section III-D.

\section{B. Available Channels with Full Neighboring Channel Blindness}

In the first case, a PP does not scan any neighboring channels at all.

In general, the number of available channels is a function of the amount of offered traffic in the cell. The average amount of traffic per RFP gives us the probability of a number of occupied channels. With that, we can find the probability of a number of available channels. We assume Poisson arrivals of calls from a very large number of users. The number of channels in use (occupied channels $C h_{\circ}$ ) at the RFP can be found with the Erlang $B$ state formula [12]

$$
\operatorname{Pr}\left\{C h_{o}=n\right\}=\frac{\frac{A_{\mathrm{RFP}}^{n}}{n !}}{\sum_{i=0}^{S_{t}} \frac{A_{\mathrm{RFP}}^{i}}{i !}} .
$$

In (1), $S_{t}$ is the total number of time slots and $A_{\mathrm{RFP}}$ the amount of traffic at the RFP. It is assumed here that callers act independently.

The PP locks onto one of the occupied channels. Of the other occupied channels, two can be in the neighboring time slots. If they are in such a neighboring time slot, they do not decrease channel availability because these channels cannot be used by the PP anyway.

We take the number of occupied channels at the RFP to be $C h_{o}$. The number of blocked time slots as seen by the PP is defined as the difference between the total time slots and the available time slots $\left(S_{t}-S_{a}\right)$ and is given by

$$
S_{t}-S_{a}=C h_{o}+2-S_{n o} .
$$

$S_{n o}$ is the number of occupied neighboring time slots and can be either two, one, or zero. The number of blocked time
TABLE I

Occupied Neighboring (Blind) Time Slots

\begin{tabular}{c|c|c}
$\begin{array}{c}\text { occupied } \\
\text { neighbouring } \\
\text { time slots } S_{n o}\end{array}$ & $\operatorname{Pr}\left\{S_{n o}\right\}$ & $\begin{array}{c}\text { available } \\
\text { time slots } \\
S_{a}\end{array}$ \\
\hline \hline 2 & $p_{1} p_{2}$ & $S_{t}-C h_{o}$ \\
\hline 1 & $2 p_{1} q_{2}$ & $S_{t}-C h_{o}-1$ \\
\hline 0 & $1-p_{1} p_{2}-2 p_{1} q_{2}$ & $S_{t}-C h_{o}-2$ \\
\hline
\end{tabular}

slots can be equal to $C h_{O}, C h_{O}+1$, or $C h_{O}+2$, depending on the number of occupied neighboring slots. To find out how many neighboring slots are occupied, we will assume that all the occupied channels are distributed uniformly over the time slots. The first occupied channel is the one the PP is locked to. Now $C h_{o}-1$ occupied channels remain to fill the other time slots. The probability that the first of the neighboring time slots is occupied is

$$
p_{1}=\frac{C h_{o}-1}{S_{t}-1} .
$$

The conditional probability that the second neighboring time slot will be occupied when the first slot already is occupied is

$$
p_{2}=\frac{C h_{o}-2}{S_{t}-2} \text {. }
$$

This is simply the number of remaining occupied channels divided by the number of remaining time slots. Now $q_{i}$ is defined as $1-p_{i}$, the probability of the event not happening. The probability that both neighboring time slots will be occupied is $p_{1} p_{2}$. The probability that only one of them will be occupied is $2 p_{1} q_{2}$. Finally, the probability that neither of the neighboring slots will be occupied is $1-p_{1} p_{2}-2 p_{1} q_{2}$. The results are summarized in Table I. The first column gives the three possibilities for the neighboring time slots. The second column shows the probabilities of these possibilities. The number of available time slots is presented in the last column.

The probability of having a number of time slots available can be established

$$
\begin{aligned}
\operatorname{Pr}\left\{S_{a}=n\right\}= & \operatorname{Pr}\left\{C h_{o}=S_{t}-n \wedge S_{n o}=2\right\} \\
& +\operatorname{Pr}\left\{C h_{o}=S_{t}-n-1 \wedge S_{n o}=1\right\} \\
& +\operatorname{Pr}\left\{C h_{o}=S_{t}-n-2 \wedge S_{n o}=0\right\} \\
& \text { for } 0 \leq n<S_{t}-3 .
\end{aligned}
$$

Of course, the number of available time slots $S_{a}$ cannot be larger than nine (in our system with $S_{t}=12$ time slots). The time slot with the locked channel is never available. The two neighboring time slots are never available in our model in which we scan only one frame from each multiframe.

In this first model, the PP does not scan any neighboring channel, and the probability of a number of available channels $C h_{a}$ is the same as the probability of the appropriate number of available time slots

$$
\operatorname{Pr}\left\{C h_{a}=10 \cdot n\right\}=\operatorname{Pr}\left\{S_{a}=n\right\}, \quad \text { for } 0 \leq n<S_{t}-3 \text {. }
$$

For all values other than multiples of ten the probability is zero. The results of (6) are shown in Fig. 3. 


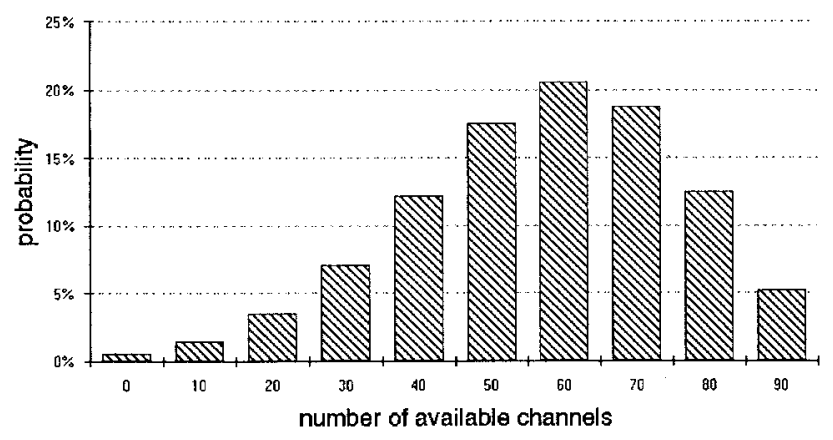

Fig. 3. The number of available channels on an RFP with 5 Erlangs traffic, when no neighboring channels are scanned.

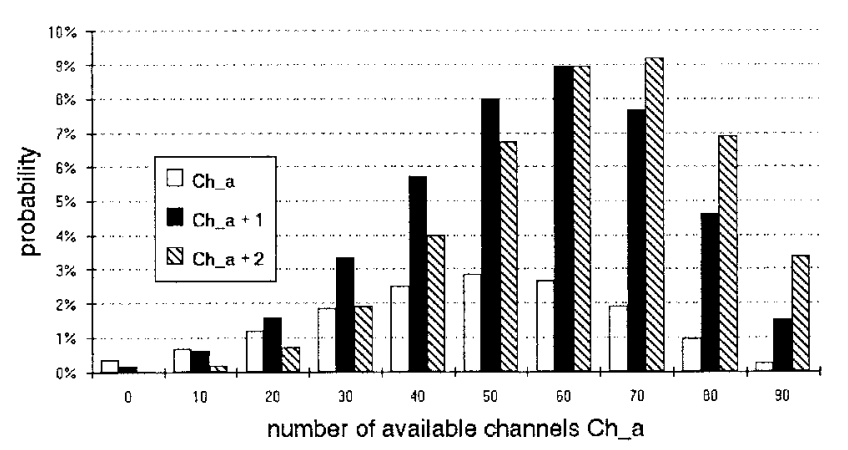

Fig. 4. The number of available channels on an RFP with 5 Erlangs traffic, when two neighboring channels are scanned.

\section{Available Channels with Partial Neighboring Channel Blindness}

The second case assumes the PP to scan the two channels immediately neighboring to the locked channel. The number of available channels is the number of available time slots times the number of carriers plus the number of available neighboring channels. This last term can be either zero, one, or two because for each neighboring time slot not occupied, one neighboring channel is available. We find

$$
\begin{aligned}
\operatorname{Pr}\left\{C h_{a}=n \cdot 10\right\} & =\operatorname{Pr}\left\{C h_{o}=S_{t}-n \wedge S_{n o}=2\right\} \\
\operatorname{Pr}\left\{C h_{a}=n \cdot 10+1\right\} & =\operatorname{Pr}\left\{C h_{o}=S_{t}-n-1 \wedge S_{n o}=1\right\} \\
\operatorname{Pr}\left\{C h_{a}=n \cdot 10+2\right\} & =\operatorname{Pr}\left\{C h_{o}=S_{t}-n-2 \wedge S_{n o}=0\right\} \\
\operatorname{Pr}\left\{C h_{a}=\text { other }\right\} & =0, \quad \text { for } 0 \leq n<S_{t}-3 .
\end{aligned}
$$

The number of available channels ranges from $0-92$, not all values included.

In Fig. 4, results are given for a system with this partial blindness. The results are grouped per number of available complete time slots. Each group of three bars totals to give the probability of the appropriate number of available complete time slots in Fig. 3. The separate bars are for the possibilities of zero, one, or two of the neighboring channels being available. The legend indicates the possibility each bar stands for. The number on the horizontal axis should be increased by zero, one or two to find the total number of available channels for which the probability is given.

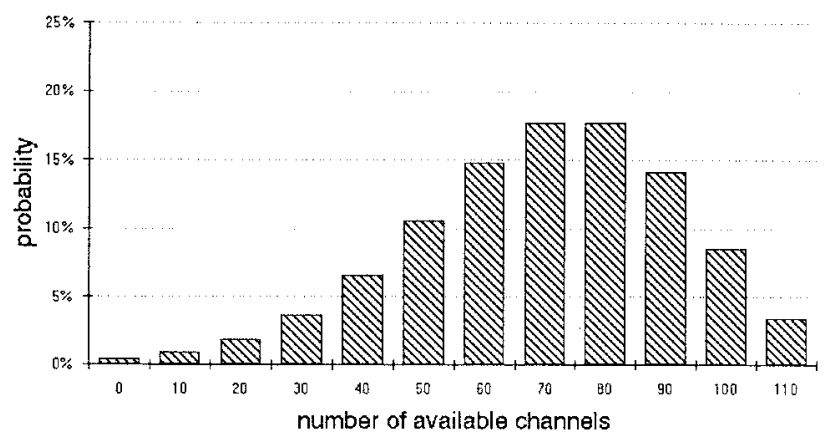

Fig. 5. The number of available channels on an RFP with 5 Erlangs traffic, when all neighboring channels are scanned.

\section{Available Channels Without Neighboring Channel Blindness}

When we consider a PP that is able to scan all channels, the function for the probability of a number of available channels is much simpler. The PP can see a maximum of 110 available channels (with the system as specified) because it does not scan any channels in the time slot of the channel it is locked on.

In this third case, the number of time slots available is

$$
S_{a}=S_{t}-C h_{o}
$$

Here, $S_{a}$ is the number of available time slots, $S_{t}$ is the total number of time slots, and $C h_{o}$ is the number of occupied channels. The probability of having a number of time slots available is

$\operatorname{Pr}\left\{S_{a}=n\right\}=\operatorname{Pr}\left\{C h_{o}=S_{t}-n\right\}, \quad$ for $0 \leq n<S_{t}-1$.

All the frequency channels of each available time slot can be accessed, so the probability of a number of available channels is

$$
\operatorname{Pr}\left\{C h_{a}=n \cdot 10\right\}=\operatorname{Pr}\left\{S_{a}=n\right\}, \quad \text { for } 0 \leq n<S_{t}-1 .
$$

Here, $C h_{a}$ is the number of available channels. The results given in Fig. 5 are actually the same as those for an Erlangs 12 system, where the possibility of 12 time slots (equivalent to 120 channels) being available has been cut off.

The difference between Figs. 3 and 4 is clear. For each number of completely available time slots, the probability does not change. In Fig. 4, e.g., the probabilities for 60-62 add to the probability of 60 free channels in Fig. 3. There is a slight overall improvement in the number of available channels in Fig. 4, due to the fact that two extra channels can be scanned. When the PP can scan all channels, more become available, as is indicated in Fig. 5. From here on, however, unless otherwise mentioned, the full blindness scheme will be used.

\section{E. Resource Blocking}

When a PP tries to set up a call and finds that it is not possible because no channel is available at the RFP, this is called "resource blocking." The probability that this will happen can easily be calculated from the above models. In Fig. 6, results are given. 


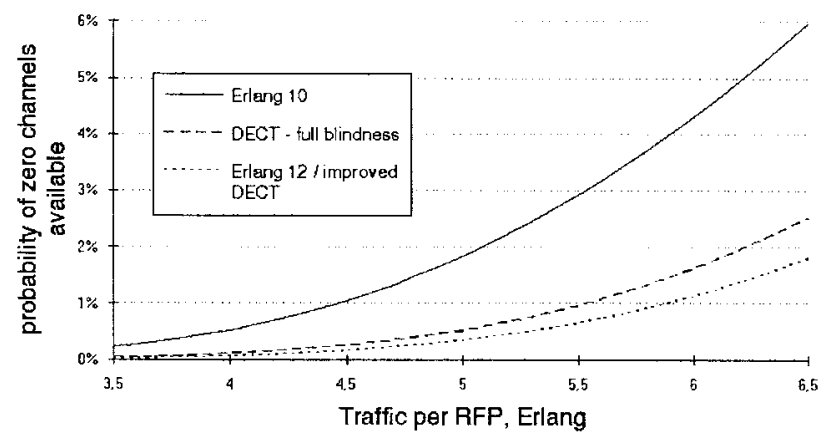

Fig. 6. The probability that no channel is available at an RFP.

The middle curve in Fig. 6 shows the result for a DECT system with full blindness. The bottom line is for an Erlangs 12 system, but also for both the two other DECT methods: partial blindness and full scanning. The top line is for an Erlangs 10 system. Implementation of a system with partial blindness is not expected to be much more complicated than a system with full neighboring channel blindness, yet an increase in blocking performance can result, as can be seen in the graph.

These results are only valid for a single-RFP system. However, when a PP cannot set up a call at the selected RFP, DECT allows the PP to try at another RFP (which must be close enough to provide acceptable carrier power at the receiver). In a system with a number of RFP's close enough to each other, the resource blocking would be greatly reduced. This is the improvement that is seen when, e.g., an Erlangs 12 system with 5 Erlangs traffic load is replaced by an Erlangs 24 system with 10 Erlangs traffic, when two RFP's are within reach.

\section{CARRIER POWER}

Because a PP has to select the RFP to connect to, we implement the RFP-selection mechanism in the model. Two different models are considered. First, however, we describe a model for the received power. A PP receives signal power from one or more RFP's. In general, this power is called rsp. The power that a PP receives from the RFP to which it is connected (i.e., the desired power) is called carrier power.

\section{A. Received Signal Power}

Signal power that is received from a distant transmitter is affected due to the following:

- antenna type and orientation;

- multipath fading;

- shadowing;

- "large-scale" path loss.

When a portable telephone is used, the antenna orientation will vary considerably. However, for the sake of simplicity we do not include a varying antenna effect into our model. We assume that the influence of the antenna type and orientation is constant. The other three effects are described in [13]. We use a model where the rsp is only influenced by the large scale path loss and the shadowing effects. A margin is added to the required CIR to compensate $99 \%$ (in time) of all multipath effects.
TABLE II

Path Loss and Transition Parameters

\begin{tabular}{|c|c|c|}
\hline $\begin{array}{l}\text { distance } \\
(\mathrm{m})\end{array}$ & $\begin{array}{c}\text { varying } \\
\text { parameters }\end{array}$ & $\begin{array}{c}\text { constant } \\
\text { parameters }\end{array}$ \\
\hline $1<d<20$ & $\alpha=3.0 \quad \beta=30$ & $\alpha=3.5$ \\
\hline $20<d \leq 40$ & $\alpha=6.0 \quad \beta=-9$ & and \\
\hline $40<d$ & $\alpha=12.0 \quad \beta=-105$ & $\beta=30$ \\
\hline
\end{tabular}

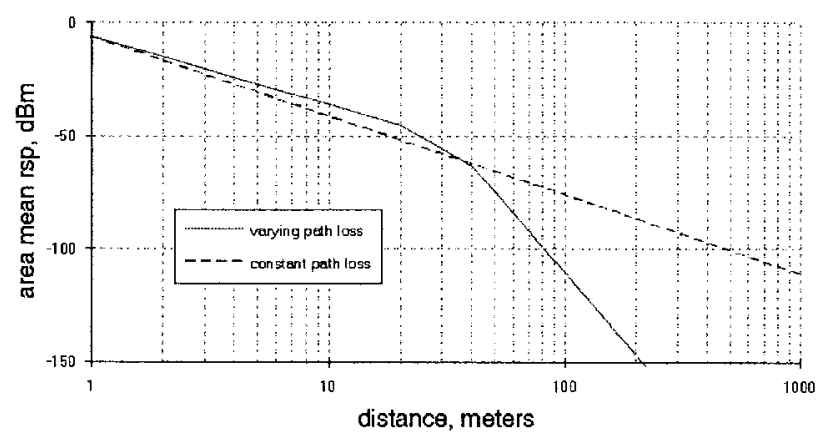

Fig. 7. The area mean rsp as a function of the distance from the transmitter.

When a receiver is at a distance $d(\mathrm{~m})$ from a transmitter, the area mean rsp ( $\overline{\overline{\mathrm{rsp}}}$, in $\mathrm{dBm})$ is

$$
\overline{\overline{\mathrm{rsp}}}(d)=P_{t}-(\beta+10 \alpha \log d), \quad \text { for } d \geq 1 \mathrm{~m} \text {. }
$$

This is the power when only large-scale attenuation is taken into account. $P_{t}$ is the transmitted power in $\mathrm{dBm}, \alpha$ the path loss parameter or decay index, and $\beta$ the parameter for the transition of transmitted power to power at one meter distance. The signal has been averaged over a large area to give this result.

For an indoor environment, we can assume the path-loss parameter $\alpha$ to vary with the distance from the transmitter. Thus, we can incorporate features like walls and floors in the model. An environment model where the path-loss parameter does not change with distance is given for comparison. This is a model that can be used for outdoor systems. Values from [14] are used in Table II for the model with varying parameters. The model with fixed parameters starts at the same rsp at 1 $\mathrm{m}$ from the antenna, but gives constant attenuation over the complete distance range.

In Fig. 7, the value of $\overline{\overline{\text { rsp }}}$ is plotted against the distance. In our calculations, the model with varying parameters is used. Even though cells with a 20-m diameter are used, in our model interference from a larger distance will influence the rsp, making the tail of the curve important.

Another aspect of the radio environment that we incorporate in this model is shadow fading. There may be objects in the path of the radio waves that attenuate the signal power. We assume that the result of this fading is a lognormal distribution of rsp, with the area mean rsp as the arithmetic mean [15]. As mentioned in Section II, the standard deviation, or spread, of the shadow fading is taken to be constant $(8 \mathrm{~dB})$ for a distance larger than $10 \mathrm{~m}$ from the transmitter. The spread will increase linearly with the distance up to $10 \mathrm{~m}$ from the transmitter. To keep the calculations manageable, we assume that shadow fading is uncorrelated in time and space. 


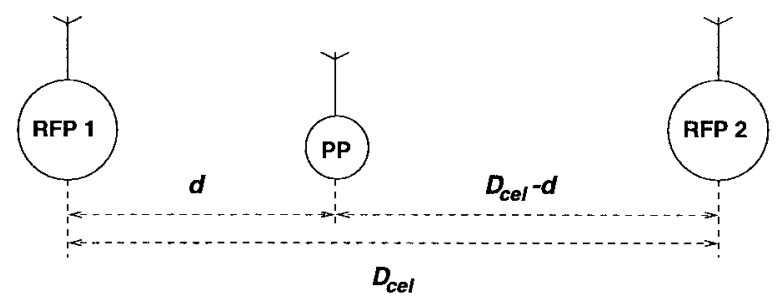

Fig. 8. The PP is on a straight line between the two RFP's that are a distance $D_{\text {cel }}$ from each other.

When we incorporate lognormal shadow fading in our model, the rsp has the following probability density function (pdf):

$$
\begin{aligned}
\operatorname{Pr} d\{\mathrm{rsp}=\operatorname{rsp} \mid d\}= & \frac{1}{\sqrt{2 \pi} \cdot \sigma} \exp \left(\frac{-(\mathrm{rsp}-\overline{\overline{\mathrm{rsp}}}(d))^{2}}{2 \sigma^{2}}\right) \\
& \text { for } d \geq 1 \mathrm{~m} .
\end{aligned}
$$

Here, $\sigma$ stands for the standard deviation of the shadow fading: the fading spread, given in decibels. The local mean rsp is called rsp throughout this paper, without the overbar to indicate local area averaging. It is given in $\mathrm{dBm}$, as is the area mean rsp. The distance to the transmitter $(d)$ is an input parameter for the pdf

Noise power can be an important factor when measuring received power strength. However, we assume the noise power to be far below the carrier power level. We therefore do not consider noise in the carrier power models.

\section{B. Selecting an RFP}

The PP moves within the system. It connects to the best RFP it can find, which is the strongest one that has a channel available. In this section we concern ourselves with the strongest RFP. We discuss two models: one where the PP can select one of two RFP's, another where three RFP's are available.

1) Selecting from Two RFP's: We place the $\mathrm{PP}$ on a straight line between two RFP's, as depicted in Fig. 8. The two RFP's are exactly one-cell diameter $\left(D_{c e l}\right)$ apart. The PP is at a distance $d$ from one RFP. Consequently, it is at a distance $D_{c e l}-d$ from the other. This does not hold for a twodimensional (2-D) system, but this approximation appeared to be accurate enough for our purpose. The location of the PP will be looked at as if it were in a 2-D cell.

If the PP is in the cell of $\mathrm{RFP}_{1}$, then the distance $d$ to $\mathrm{RFP}_{1}$ cannot be larger than $D_{c e l} / 2$. We know the pdf's for the rsp for both RFP's at their respective distances, from (12). We use them to calculate the probability of a level of carrier power.

We take the placement probability density of the PP to be uniform within a circular cell. The probability density of the PP being at a distance $d$ (in $\mathrm{m}$ ) from $\mathrm{RFP}_{1}$ is

$$
\operatorname{Pr} d\{d=x\}=\frac{8 x}{D_{c e l}^{2}}, \quad \text { for } x<\frac{D_{c e l}}{2} .
$$

The PP selects the RFP with the strongest signal. When it receives signal power $c$ from $\mathrm{RFP}_{1}$ and less from $\mathrm{RFP}_{2}$, it selects $\mathrm{RFP}_{1}$. The carrier power then is $c \mathrm{dBm}$. If the power levels are reversed, the PP will select $\mathrm{RFP}_{2}$.

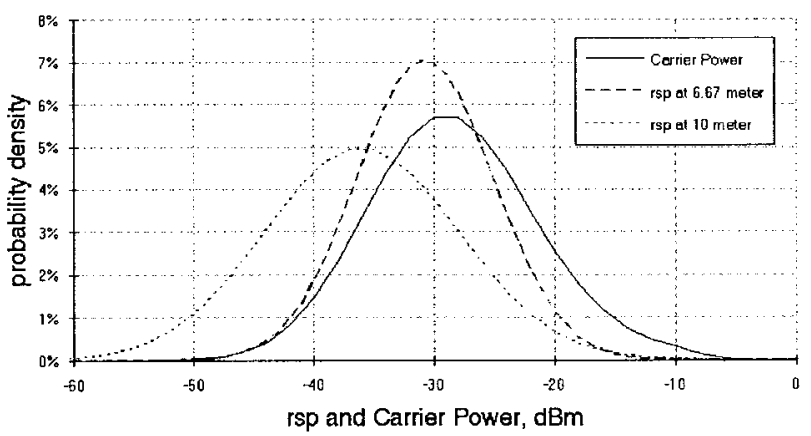

Fig. 9. Best rsp (carrier power) from two RFP's, together with rsp's for cell boundary $(10 \mathrm{~m})$ and average distance $(6.67 \mathrm{~m})$.

We now define a short notation for the probability density and cumulative pdf of the rsp

$$
\begin{aligned}
& P(\operatorname{rsp}, x)=\operatorname{Pr} d\{\mathrm{rsp}=\operatorname{rsp} \mid x\} \\
& C(\operatorname{rsp}, x)=\operatorname{Pr}\{\operatorname{rsp}<\operatorname{rsp} \mid x\} .
\end{aligned}
$$

Here, $x$ stands for the distance of the PP to the respective RFP. We assume that the pdf's for carrier power are identical for all RFP's and that the signals are uncorrelated.

The conditional probability density that at a certain distance $d$ (in $\mathrm{m}$ from $\mathrm{RFP}_{1}$ ) we will find rsp of strength $c(\mathrm{dBm})$ from $\mathrm{RFP}_{1}$ and less from $\mathrm{RFP}_{2}$ is

$$
\begin{aligned}
\operatorname{Pr} & d\left\{\mathrm{CP}=c \wedge \mathrm{RFP}_{1} \text { chosen } \mid d\right\} \\
\quad & \operatorname{Pr} d\{\mathrm{rsp}=c \mid d\} \cdot \operatorname{Pr}\left\{\mathrm{rsp}<c \mid D_{c e l}-d\right\} \\
& \equiv P(c, d) \cdot C\left(c, D_{c e l}-d\right) .
\end{aligned}
$$

This product gives us the probability of carrier power $\mathrm{CP}=c$ when we know in advance that the PP will select $\mathrm{RFP}_{1}$.

When we add to this the conditional probability density that we will receive rsp $c$ from $\mathrm{RFP}_{2}$ and less from $\mathrm{RFP}_{1}$, we find the total probability density of carrier power $c$ at the given distance

$$
\begin{aligned}
\operatorname{Pr} d\{\mathrm{CP}=c \mid d\}= & P(c, d) \cdot C\left(c, D_{c e l}-d\right) \\
& +P\left(c, D_{c e l}-d\right) \cdot C(c, d) .
\end{aligned}
$$

Still, $d$ stands for the distance from $\mathrm{RFP}_{1}$.

Integrating (16) over the distance yields the unconditional pdf for the carrier power for a PP at an arbitrary (unknown) place

$$
\begin{aligned}
\operatorname{Pr} d\{\mathrm{CP}=c\}= & \int_{0}^{D_{c e l} / 2} \operatorname{Pr} d\{\mathrm{CP}=c \mid d=x\} \\
& \cdot \operatorname{Pr} d\{d=x\} d x .
\end{aligned}
$$

The result for this method is shown, along with two curves for verification, in Fig. 9. The pdf's for rsp at two fixed distances as calculated with (12) are given-one for the average distance of the PP from $\operatorname{RFP}_{1}(6.67 \mathrm{~m})$ and one for the cell radius $(10 \mathrm{~m})$. The third curve is the best power from the twoRFP model: the carrier power. (Note that the transmitted power $P_{T}$ is $24 \mathrm{dBm}$.) The probability of exceeding a certain level of carrier power is always higher than the same probability for the rsp at the cell boundary. Also, it is nearly always higher than 


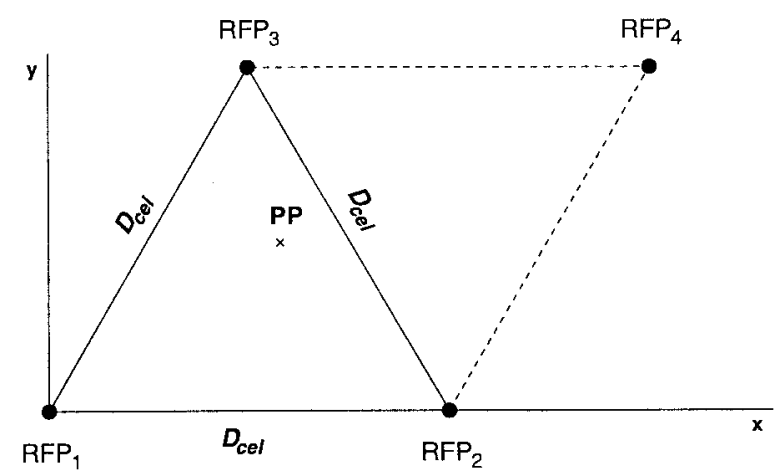

Fig. 10. The placement of the three nearest RFP's around a PP. The PP can be anywhere within the triangle.

the probability of exceeding the rsp at the average distance. These findings reflect the selection of the best of two RFP's.

When a PP cannot set up a call at the first RFP (no available channels, no channels good enough), it reverts to the next best RFP (if any). We then need the carrier power from that RFP. The method reported above can also be applied when we need to know the power, given the fact that we selected the second best RFP when the call setup did not succeed at the best RFP.

2) Selecting from Three RFP's: The model with only two RFP's is not very realistic: PP's are not always on a straight line between two RFP's. We now look at a better 2-D model.

The hexagonal cell shape is very effective when packing a number of cells into a certain area. The RFP's are placed according to such a hexagonal cell shape and form a grid as indicated in Fig. 10. We will investigate the area between RFP's 1-3. Only these three RFP's will be taken into account. When a PP is near a border, say between $\mathrm{RFP}_{2}$ and $\mathrm{RFP}_{3}$, there will be an $\mathrm{RFP}_{4}$ about as far away from the PP as $\mathrm{RFP}_{1}$. $\mathrm{RFP}_{1}$ and $\mathrm{RFP}_{4}$ are the two farthest RFP's. The influence of $\mathrm{RFP}_{1}$ will be small, and the same holds for $\mathrm{RFP}_{4}$. When the PP is at any other place within the triangle, $\mathrm{RFP}_{1}$ will influence the carrier power much more than $\mathrm{RFP}_{4}$. We will neglect the influence of $\mathrm{RFP}_{4}$, and any other RFP outside the triangle, altogether.

We give each RFP a pair of Cartesian coordinates to indicate their positions. For each RFP $i$, we thus have $x_{\mathrm{RFP}_{i}}$ and $y_{\mathrm{RFP}_{i}}$. The PP which is at $(x, y)$ is a distance

$$
d_{\mathrm{RFP}_{i}}=\sqrt{\left(x_{\mathrm{RFP}_{i}}-x\right)^{2}+\left(y_{\mathrm{RFP}_{i}}-y\right)^{2}}
$$

from $\mathrm{RFP}_{i}$. We assume that the PP has a uniform spatial distribution, so that the probability of $(x, y)$ is constant.

Again, we use (14) for the probability and cumulative pdf's. Now the probability of a carrier power of $c$ is the probability that one RFP offers $c$ while the other two offer less

$$
\begin{aligned}
& \operatorname{Pr} d\{\mathrm{CP}=c \mid x, y\} \\
&= P\left(c, d_{\mathrm{RFP}_{1}}\right) \cdot C\left(c, d_{\mathrm{RFP}_{2}}\right) \cdot C\left(c, d_{\mathrm{RFP}_{3}}\right) \\
&+P\left(c, d_{\mathrm{RFP}_{2}}\right) \cdot C\left(c, d_{\mathrm{RFP}_{1}}\right) \cdot C\left(c, d_{\mathrm{RFP}_{3}}\right) \\
&+P\left(c, d_{\mathrm{RFP}_{3}}\right) \cdot C\left(c, d_{\mathrm{RFP}_{1}}\right) \cdot C\left(c, d_{\mathrm{RFP}_{2}}\right) .
\end{aligned}
$$

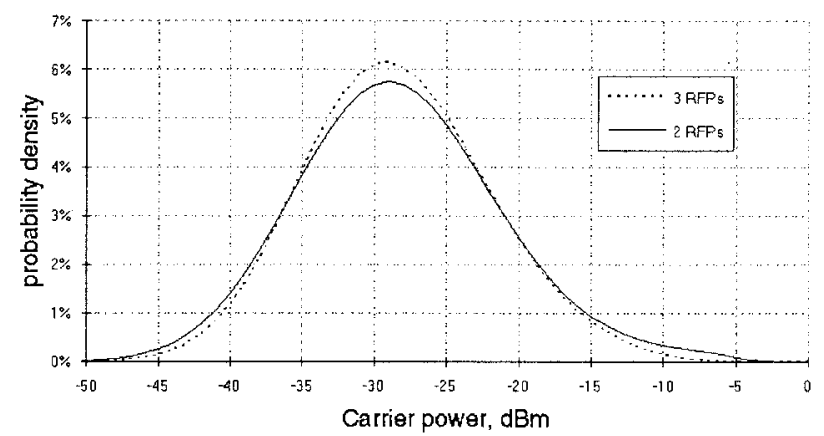

Fig. 11. PDF's for carrier power, shown for varying path loss. The best power of two or three RFP's is selected.

This remains to be averaged over all values of $x$ and $y$ that lie within the triangle

$$
\begin{gathered}
\operatorname{Pr} d\{\mathrm{CP}=c\} \\
=\frac{1}{O_{\text {triangle }}} \int_{0}^{l_{1}} \int_{l_{2}}^{l_{3}} \operatorname{Pr} d\{\mathrm{CP}=c \mid x, y\} d x d y \\
\quad \text { with } l_{1}=\sqrt{3} D_{c e l} / 2 ; \quad l_{2}=y / \sqrt{3} \\
\quad l_{3}=D_{c e l}-y / \sqrt{3} .
\end{gathered}
$$

Here, $O_{\text {triangle }}$ is the area over which we integrated. One divided by this value is the probability density of being at point $(x, y)$, which is constant for all $x$ and $y$. The algorithm can also be applied when looking for the power from the second best RFP, except that then the probability should be used that one RFP will offer better power than the given value.

The pdf's of carrier power for a $D_{\text {cel }}$ of $20 \mathrm{~m}$ for both the 2 and 3 RFP model are shown in Fig. 11. It is reassuring to see how similar the results are for the two different methods. They show that although the distance to the nearest RFP can be larger in the 3 RFP model, the higher number of RFP's to select from compensates the higher average attenuation loss.

\section{INTERFERENCE POWER}

A method to calculate the cochannel interference power is described in this section. This is the undesired power that a PP receives from other users that are communicating on the same channel. Although these users usually are at a large distance, their power can cause interference.

We calculate a pdf for the rsp on a channel. With all the pdf's for all the available channels, we need a method of selecting the best channel. This will lead us to a pdf for interference power. In this model, all the RFP's are in perfect frame synchronization. The DECT standard requires close synchronization of cooperating RFP's.

\section{A. Received Signal Power on a Single Channel}

In the following discussion, we must keep in mind that the interference power a PP experiences is transmitted by RFP's. When we are speaking of users in the interference context, the RFP's to which the users (PP's) are connected are intended. The power from those RFP's and the distance to those RFP's are the important parameters. For the sake of 
simplicity, however, we will keep on referring to the "users" from which interference is experienced.

Another item to be considered is the noise. The power from noise is not included in the derivations in this section. When noise should be reckoned with, i.e., when interference power becomes too low, noise should be added to the rsp. The interference then is calculated on the basis of the combined received signal and noise power. If this is done, it will be explicitly mentioned along with the results, otherwise, results are for interference on the basis of rsp only, although the results may be below the noise power level.

Suppose the observed circular area is so large that enlarging it would not influence the results. All signals from outside the area should be negligible at the center of the area, which is the point under observation. The active users (spread uniformly in space) will also spread evenly over the total number of channels $\left(C h_{t}\right)$, which is the purpose of the DCS. We can calculate the number of users per channel, given the number of active users. The interference power at a certain point is the power from the active users on the best channel. Given the statistical approach, a channel will be found with active users only at the boundaries of the area, resulting in very low interference power. This does not agree with the practical behavior that PP's on one channel will equalize their mutual distances. We should constrain the PP's to smaller subareas to avoid this trap.

Taking a part of an infinitely large area, we assume that due to the even spread of the users we find a circular area within which each of the $C h_{t}$ channels is being used by exactly one user. We will take the size of the circular area such that there are $C h_{t}$ users active. At the center of this circular area an arbitrary PP wants to set up a call: this is the observed PP. Now, if one channel could be found without an active user in this area, another channel would be used by two users to keep the total number of users equal to $C h_{t}$. The only reason for this channel state can be that interference from just outside the area on the channel with no users is so strong that it is not better than other possible channels, as seen from the observed PP. Then the observed PP is not more likely to choose this empty channel than any other channel. This model with the circular area around the observed PP assumes that the DCS algorithm functions well.

Now, we need an area in which $C h_{t}$ users are active. For an area with $A$ Erlangs traffic, the probability of $C h_{t}$ active users is given by the Erlang $B$ state equation

$$
\operatorname{Pr}\left\{N_{\text {active }}=C h_{t}\right\}=\frac{\frac{A^{C h_{t}}}{C h_{t} !}}{\sum_{i=0}^{U_{\max }} \frac{A^{i}}{i !}}
$$

Here, $U_{\max }$ is the maximum number of users the RFP's in the area can simultaneously serve. To obtain an area where all channels are occupied once (with $C h_{t}=120$ active users), we need an area with roughly 120 Erlangs of traffic. With 5 Erlangs per RFP we will need, on average, 24 RFP's. These RFP's offer channels for a maximum of $U_{\max }=24 \cdot 12=288$ users at any one time. This is large enough to approximate
(21), letting $U_{\max }$ go to infinity, in which case we find the Poisson distribution

$$
\operatorname{Pr}\left\{N_{\text {active }}=C h_{t}\right\} \approx \frac{A^{C h_{t}}}{C h_{t} !} e^{-A} .
$$

What we need, however, is the probability for such an area (or, equivalently, for the total amount of traffic needed) that all $C h_{t}$ channels are occupied. We now calculate (22) for all values of $A$ keeping the number of active users fixed. Using the resulting values, we find the a posteriori probability density for the amount of traffic. This yields

$$
\operatorname{Pr} d\left\{A=a \mid C h_{t}\right\}=\frac{a^{C h_{t}}}{C h_{t} !} e^{-a}
$$

when the a priori unconditional probability (respectively, density) for the number of active users (respectively, amount of total traffic) is taken to be uniform. Equation (23) gives the probability density of an average of $a$ Erlangs traffic, when we know that $C h_{t}$ channels are occupied.

From cell traffic and size we can calculate the total area needed to provide the $a$ Erlangs of traffic. Cells are assumed to be hexagonal, so

$$
O_{c e l}=\frac{1}{2} \sqrt{3} D_{c e l}^{2} .
$$

Let us call the total area in which each channel is occupied by one user $O_{1}\left(\mathrm{~m}^{2}\right)$. The radius of this area is $R_{1}(\mathrm{~m})$. For an area with $a$ Erlangs traffic, we define $O_{1}$ and $R_{1}$

$$
O_{1}=\frac{a}{A_{R} F P} O_{c e l} \quad R_{1}=\sqrt{O_{1} / \pi} .
$$

Here, $A_{\mathrm{RFP}}$ is the average traffic per RFP. Now, on each channel we have one user that is within $R_{1} \mathrm{~m}$ from the observed PP. The place of the user is assumed to have a uniform probability distribution. Thus, the probability density for a distance $d_{u}$ of an arbitrary user can be found as

$$
\operatorname{Pr} d\left\{d_{u}=d\right\}=\frac{2 d}{R_{1}^{2}} .
$$

However, we are not interested in users that are connected to the RFP the observed PP is using. Channels already in use at that RFP cannot be used by the observed PP anyway. We can modify the carrier power calculations in such a way that we find the probability that an arbitrary PP is not using $\mathrm{RFP}_{w}$. $\mathrm{RFP}_{w}$ is the wanted RFP, where the observed PP wants to set up a call. We then get

$$
P_{\mathrm{RFP}_{w}}=\operatorname{Pr}\left\{\operatorname{RFP}_{w} \text { not selected } \mid d_{u}=d\right\} .
$$

When we multiply this probability with the probability density of (26), we find the pdf of an arbitrary user being at distance $d$ and not connected to $\mathrm{RFP}_{w}$

$$
\operatorname{Pr} d\left\{d_{u, n}=d\right\}=\operatorname{Pr} d\left\{d_{u}=d\right\} \cdot P_{\mathrm{RFP}_{w}} .
$$

This value has to be normalized to give a total probability of one.

If one user is present on each channel within the area $O_{1}$, we can use the pdf for rsp from one user as given in (12). We must now integrate the pdf for rsp over the circular area, using 
the probability that a user is at distance $d$ and not connected to $\mathrm{RFP}_{w}$

$$
\begin{aligned}
P_{\mathrm{rsp}_{O 1}}(\mathrm{rsp})= & \operatorname{Pr} d\left\{\mathrm{rsp}_{O 1}=\mathrm{rsp}\right\} \\
= & \int_{0}^{R_{1}} \operatorname{Pr} d\left\{\mathrm{rsp}_{O 1}=\mathrm{rsp} \mid d\right\} \\
& \cdot \operatorname{Pr} d\left\{d_{u, n}=d\right\} d d .
\end{aligned}
$$

We can now assume a second region (a concentric ring) around circular area $O_{1}$, which also accommodates $C h_{t}$ users. This region accommodates a second interferer for each channel. Its area can be noted as $\mathrm{O}_{2}$. Within this ring, the $C h_{t}$ users each select a channel, so that in total each channel is used by two users. The outer boundary is at a distance of

$$
R_{2}=\sqrt{O_{1}+O_{2}} / \pi \text {. }
$$

The probability of an arbitrary user being at a certain distance within this region is

$$
\begin{aligned}
\operatorname{Pr} d\left\{d_{u}=d\right\} & =\operatorname{Pr} d\left\{d_{u, n}=d\right\} \\
& =\frac{2 d}{R_{2}^{2}-R_{1}^{2}}
\end{aligned}
$$

safely assuming that the PP is not connected to $\mathrm{RFP}_{w}$. We can also calculate a pdf for interference power from the second region

$$
\begin{aligned}
P_{\mathrm{rsp}_{O 2}}(\mathrm{rsp})= & \operatorname{Pr} d\left\{\mathrm{rsp}_{O 2}=\mathrm{rsp}\right\} \\
= & \int_{R_{1}}^{R_{2}} \operatorname{Pr} d\left\{\mathrm{rsp}_{O 2}=\operatorname{rsp} \mid d\right\} \\
& \cdot \operatorname{Pr} d\left\{d_{u, n}=d\right\} d d .
\end{aligned}
$$

Convolving the two pdf's for interference power results in a better approximation because a second interferer is reckoned with. The assumption of one user per channel is less valid for the outer ring than for the inner circle, but it is still good enough to use for the outer ring because these interferers have less influence than the interferers in the inner circle. The pdf for the total signal power received (rsp) by a PP on an arbitrary channel becomes

$$
\begin{aligned}
& P_{\mathrm{rsp}_{O 1 \& O 2}}(\mathrm{rsp}) \\
& \quad=\operatorname{Pr} d\left\{10 \log \left(10^{\mathrm{rsp}_{O 1} / 10}+10^{\mathrm{rsp}_{O 2} / 10}\right)=\operatorname{rsp}\right\} .
\end{aligned}
$$

Equation (32) can be calculated with the convolution transformed for the logarithmic domain [16]. We will use the results of (32) in the next section to calculate the interference power on an arbitrary channel. Fig. 12 shows some results. For both the inner and outer areas, a traffic of 120 Erlangs is assumed, so the areas are fixed in size. The rsp from both areas is given in two curves. The pdf of the sum of these powers is displayed as the bold curve. This is the total rsp that a PP receives from the interferers. With this system size (cell diameter $20 \mathrm{~m}$ ), the second interferer slightly influences the overall result. A third interferer would be even further away. The influence would then be negligible.

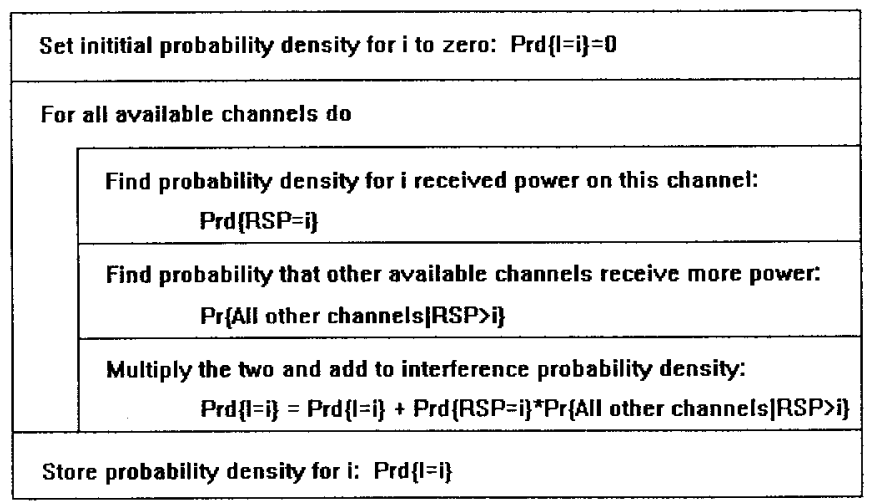

Fig. 12. Calculation of interference probability density.

\section{B. Selecting the Best Channel}

We consider the system from the viewpoint of a PP; PP's have the initiative in the DCS system. Let us imagine a PP that wants to start communicating. It finds itself in the vicinity of a certain RFP. If there are more, the PP will select the one from which it receives the strongest power. The rsp of the channel to be selected will become the interference as soon as the PP starts using the channel. Therefore, from the channels available at the RFP, the PP will select the one with the lowest rsp (in the case with noise, the PP will select the channel with the lowest rsp plus noise). For the availability of channels we can choose a model depending on the implementation of channel scanning in the PP.

When no channels are available, a call cannot be set up. We do not consider this blocking possibility here. We discuss establishing the pdf of interference power for a PP that has succeeded in a call setup.

The PP has to select one of the available channels. To be able to include a selection in our model, we have already established the pdf for the rsp of all available channels. The rsp is the power that is received at the position of our PP from the users of a channel.

We can calculate the probability that we will select a certain level of signal power. The selected rsp will then become the "interference power" (by definition). The channel we select must be the one with the lowest rsp of all. We will get the probability that all the other channels offer a higher rsp than the one we select, at a specific level of the rsp. In Fig. 12, the steps are visualized. This structure diagram describes how to find the probability density for interference power, given the number of available channels. When noise is included in the calculations, the rsp should be replaced by rsp plus noise.

We have found a pdf of the rsp for all the available channels at our RFP. We also have a probability function for the number of available channels. It now remains to find the pdf for interference power.

We can offer our imaginary PP a number of channels with corresponding pdf's for the rsp. The PP must select one, based on the rsp of all available channels. This is done in much the same way as for the carrier power: the PP selects the best available power. In this case, that means that the channel with the lowest rsp will be selected. 


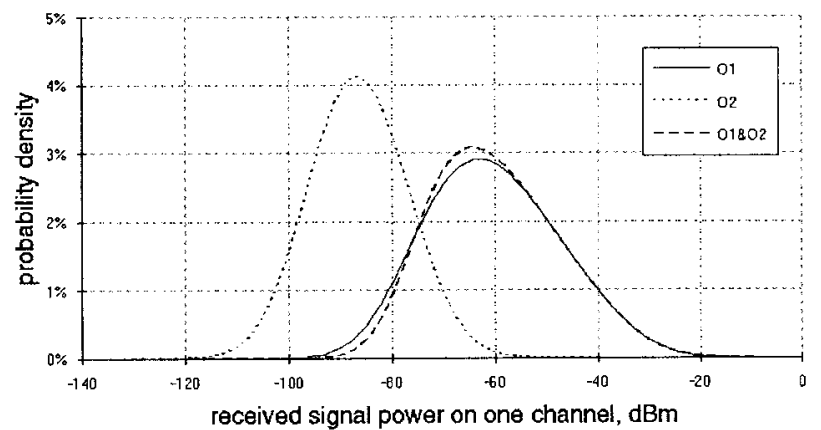

Fig. 13. Received signal power for different areas. The areas are fixed in size. No noise is included.

To calculate the probability of a level of interference power, we first need the probability density of a certain level of rsp on one channel. We find it using (32). Then, we need the probability that the rsp of all the other channels is higher. With $C h_{a}$ available channels we find

$$
\begin{aligned}
& \operatorname{Pr}\{\text { all other channels higher than } i\} \\
& \quad=\left(\operatorname{Pr}\left\{\operatorname{rsp}_{O 1 \& O 2}>i\right\}\right)^{C h_{a}-1} .
\end{aligned}
$$

Now we multiply and sum over all possibilities of channel availability

$$
\begin{aligned}
P_{I}\left(i \mid O_{1} \& O_{2}\right) \\
=\sum_{c=1}^{C h_{\max }}\left[c \cdot \operatorname{Pr}\left\{C h_{a}=c\right\} \cdot P_{\mathrm{rsp}_{O 1 \& O 2}}(i)\right. \\
\left.\quad \cdot\left(\operatorname{Pr}\left\{\operatorname{rsp}_{O 1 \& O 2}>i\right\}\right)^{c-1}\right] .
\end{aligned}
$$

The result is the pdf of the interference power, given the sizes of the circular areas. If we want to average over the possible area sizes, (34) should be integrated over the area sizes. Both areas $O_{1}$ and $O_{2}$ might be varied. However, to reduce calculation times, only area $O_{1}$ is varied in the calculations. This has a negligible impact on the results since the contribution of $\mathrm{O}_{2}$ is small, as can be seen in Fig. 13. The general equation used is

$$
\begin{aligned}
P_{I}\left(i \mid A_{\mathrm{RFP}}\right)= & \int_{O_{\min }}^{O_{\max }} \operatorname{Pr} d\left\{A=\frac{O_{1}}{O_{E r l}} \mid C h_{t}\right\} \\
& \cdot P_{I}\left(i \mid O_{1} \& O_{2}\right) d 0_{1}
\end{aligned}
$$

where (23) is used for the probability density of the area size, using the equivalence of the pdf's for traffic $A$ and area $O_{1}$. The amount of traffic is found by dividing the area $O_{1}$ by the area needed for one Erlangs $\left(O_{\mathrm{Erl}}\right)$. The size of $\mathrm{O}_{2}$ is kept constant. The interference is also calculated for a fixed inner area $O_{1}$, which holds an average traffic of 120 Erlangs. No integration is necessary then.

In Fig. 14, four curves are displayed which give the interference results for different areas. The simplest calculation was for a single area with 120 Erlangs. The most sophisticated method uses a varying inner area and a constant outer area. Here, the results are averaged over all the possibilities for the inner area. The outer area is kept constant since its impact is small (see also Fig. 13). The two other curves show effects of modifications of the method.

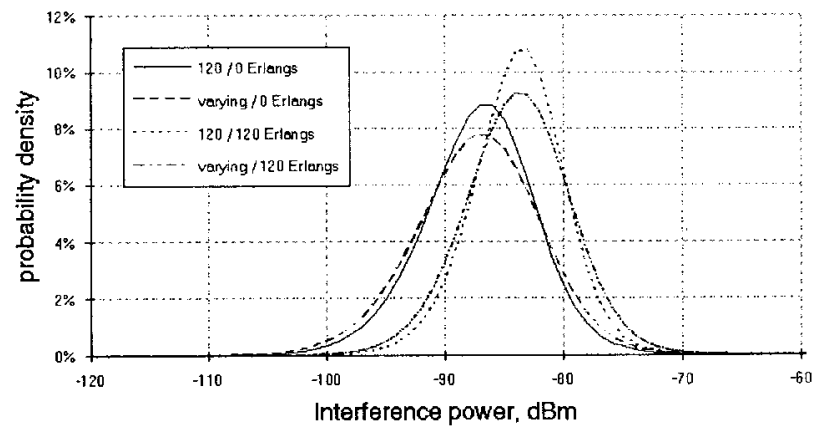

Fig. 14. Interference for different areas. The traffic used in the areas is indicated in the legend. 120/0 means: inner circle fixed at 120 Erlangs, outer layer fixed at 0 Erlangs (i.e., no outer layer). No noise is included.

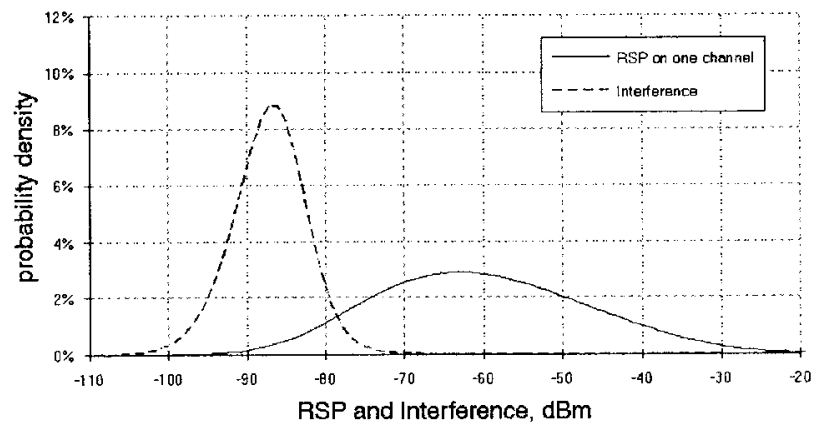

Fig. 15. RSP and interference within fixed area. $O_{1}$ was used. No noise is included.

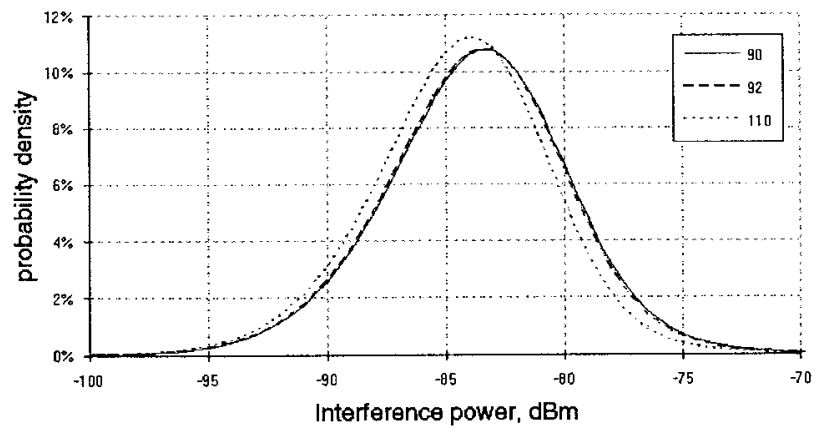

Fig. 16. Interference power for different available channel models. $\mathrm{O}_{1} \& \mathrm{O}_{2}$, both fixed. The legend indicates the maximum number of available channels. No noise is included.

Fig. 15 shows how the mathematical model selects a level of rsp to be interference: the lowest rsp values that are possible are selected.

Finally, in Fig. 16, we give the results for the different models developed for channel availability. The two additional channels hardly change the interference results. The 20-channel increase, however, has some noticeable effect for the better. It can be concluded that the channel availability model's main importance lies with the resource blocking and not with interference.

\section{CARRIER-TO-INTERFERENCE RATIO}

When both the carrier power and the interference power pdf's are known, the calculation of the CIR is a straightforward 


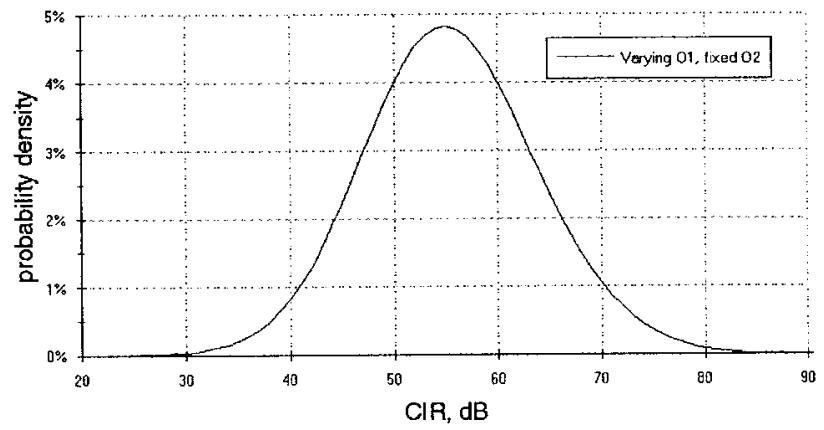

Fig. 17. PDF of CIR. $O_{1}$ is variable and $O_{2}$ is fixed. No noise was included.

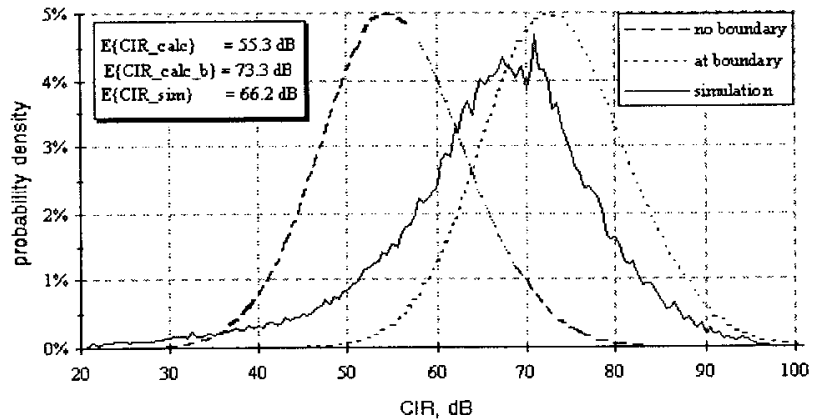

Fig. 18. PDF of CIR. Both areas $O_{1}$ and $O_{2}$ are fixed. No noise was included. Also the results for a PP at a straight boundary are given, as well as simulation results for a bounded system. The average values of the three functions are also given in a box.

matter. A convolution of the two pdf's is all that is needed

$$
\operatorname{Pr} d\{\mathrm{CIR}=r\}=\int \operatorname{Pr} d\{C=p\} \cdot \operatorname{Pr} d\{-I=r-p\} d p .
$$

CIR results are given in Fig. 17. It shows that the probability of the CIR to be below $21 \mathrm{~dB}$ is very close to zero.

A comparison with simulation results shows some differences. The curve shapes are alike, but the mean values differ, as can be seen in Fig. 18. The discrepancies are likely to be caused by differences between the system models. Most important among the differences is the fact that the simulation uses a bounded area, whereas the mathematical models assume (a part of) an infinite area. The simulation used a rectangular area containing 64 RFP's. When a system with a bounded area is investigated, it is not possible to use the circular approach of Section V. A PP can be close to the border of the system, so that all the interferers are in a semicircle around the PP. The average distance of the interferers then is larger than in our case. Interference power will be lower. For a PP at a straight boundary, it is straightforward to compute the interference power. A semicircle should then be used instead of the circular area. The results confirm that bounded systems will experience lower interference. A PP in a corner of such a system would find interferers even further away, lowering interference more. When a mathematical model would be averaged over all such possibilities, we feel that the results will be very close to the simulation results.

\section{CONCLUSIONS}

The mathematical models developed in this report appear to be satisfactory. Results for carrier power and interference power show that the logical basis of the models is solid.

The end goal, a mathematical relation for the call blocking, is already supported by the following models.

- Channel Availability Model: Depending on the implementation of a PP, a probability function can be used for the number of available channels. This model shows that for a single RFP the resource blocking using DCS is at best equal to the resource blocking using an equivalent FCA.

- Carrier Power Model: Two models were developed, giving almost equal results. In one model, two RFP's were present to select from, and in the other model, three RFP's were available.

- Interference Power Model: The developed method is used to compute the rsp from the closest user on the best channel. A second, more distant, user may also contribute to the rsp on the channels.

- The CIR can be computed by a convolution of the carrier and interference powers.

Finally, the call blocking is a function of the results of different models, which are channel (or resource) blocking, carrier blocking, and CIR blocking. From the results reported here, it can be concluded that the resource blocking is the most important factor of call blocking.

A comparison with simulation shows some differences. These are likely to be a consequence of differences in the system models. Important is the fact that the simulation only reckons with a bounded area. The mathematical model works on (part of) an infinite system.

The results are only valid when the PP can access but one RFP. What should be investigated is the correlation between the different cells. How is the traffic of bordering cells correlated? What happens if a call cannot be set up in one cell? Will the portable revert to the nearest cell? These questions still need a lot of attention and will be the subject of further research.

\section{ACKNOWLEDGMENT}

The authors would like to thank the anonymous reviewers for their valuable suggestions.

\section{REFERENCES}

[1] P. A. Raymond, "Performance analysis of cellular networks," IEEE Trans. Commun., vol. 39, no. 12, pp. 1787-1793, 1991

[2] D. C. Cox and D. O. Reudink, "A comparison of some dynamic channel assignment strategies in large-scale mobile communications systems," IEEE Trans. Commun., vol. COM-20, pp. 190-195, 1972.

[3] S. Fukumoto et al., "Dynamic channel allocation using a channel framework for traffic congestion in a highway micro cellular system," in PIMRC'94, Hague, The Netherlands, 1994, pp. 164-168.

[4] M. Frodigh, "Bounds on the optimum performance of dynamic channel assignment algorithms in 1-dimensional cellular radio systems," Radio Communications Systems, Royal Institute of Technology, Sweden, Rep. TRITA-RCS-9204, Oct. 27, 1992.

[5] _ "Maximum packing channel allocation for 1-dimensional cellular radio systems," Radio Communications Systems, Royal Institute of Technology, Sweden, Rep. TRITA-TTT-9112, Oct. 16, 1991.

[6] D. C. Cox and D. O. Reudink, Microwave Mobile Communications, W. C. Wakes, Jr., Ed. New York: Wiley, 1974, ch. 7, pp. 545-622. 
[7] B. Bout, D. Sparreboom, F. Brouwer, and R. Prasad, "A mathematical model for dynamic channel selection in conformity with the digital European cordless telecommunications standard," in Proc. PIMRC'93, Yokohama, Japan, pp. 547-551.

[8] J. B. Punt, D. Sparreboom, and F. Brouwer, "Mathematical models for the analysis of dynamic channel selection for indoor mobile wireless communication systems" in Proc. PIMRC'94, Hague, The Netherlands, Sept. 18-22, 1994, pp. 1081-1085.

[9] Radio Equipment and Systems: Digital Enhanced Cordless Telecommunications, reference document, European Telecommunications Standards Institute, ETR 015.

[10] Radio Equipment and Systems: Digital Enhanced Cordless Telecommunications, Common Interface Parts 1-3: Overview, Physical Layer and Medium Access Control Layer, European Telecommunications Standards Institute, ETR 300 175-1, ETR 300 175-2, ETR 300 175-3.

[11] Radio Equipment and Systems: Digital Enhanced Cordless Telecommunications, Common Interface: Services and Facilities Requirements Specification, European Telecommunications Standards Institute, ETR 043.

[12] M. Schwartz, Telecommunication Networks, Protocols, Modeling and Analysis. New York: Addison-Wesley, 1987, pp. 54-55.

[13] J.-P. Linnartz, Narrowband Land-Mobile Networks. Norwood, MA: Artech, 1993, pp. 29-30.

[14] R. C. Bernhardt, "The effect of path loss models on the simulated performance of portable radio systems," in Proc. IEEE GLOBECOM'89, 1989.

[15] H. Hashemi, "Impulse response modeling of indoor radio propagation channels," IEEE J. Select. Areas Commun., vol. 11, no. 7, pp. 967-978, 1993.

[16] J. B. Punt and D. Sparreboom, "Summing received signal powers with arbitrary probability density functions on a logarithmic scale," Wireless Personal Communications 3, pp. 215-224, 1996.

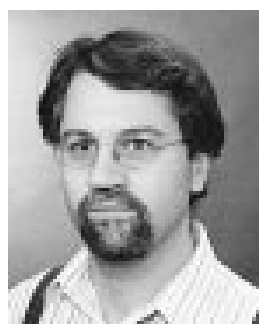

Jan B. Punt received the M.Sc. (Ir.) degree in 1994 from the Delft University of Technology, Delft, The Netherlands.

$\mathrm{He}$ is participating in the European FRAMES research project in Delft, which is carried out under the ACTS umbrella, where he continues to work on mobile telecommunications.

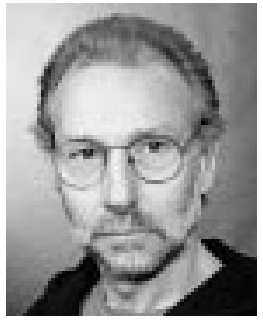

Dirk Sparreboom received the M.Sc. (Ir.) degree in electrical engineering in 1970 from Delft University of Technology, Delft, The Netherlands.

He worked on modeling of biological systems until 1983. Then, he joined the Faculty of Information Technology and Systems, Delft University of Technology, to work in the Telecommunications and Traffic-Control Systems Group on data communication protocols. His current main research subject is network aspects of mobile communication. He is in charge of lectures on data communications and networks and performance analysis.

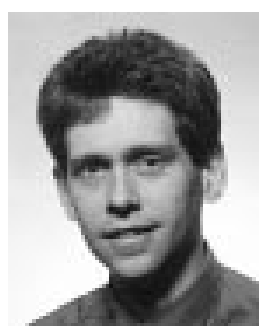

Frank Brouwer was born in Nijmegen, The Netherlands, on December 29, 1965. He received the M.Sc. degree in electrical engineering in 1989 from the Delft University of Technology, The Netherlands.

In 1991, he joined Ericsson Business Mobile Networks as a Systems Engineer. Since 1996, he has been at Ericsson as a Strategic Engineer. His field of expertise is the air-interface performance of cordless systems. He considers both link quality and system capacity, with a focus on DECT-based systems.

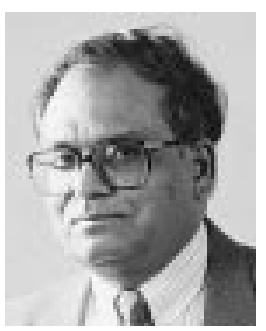

Ramjee Prasad (M'88-SM'90) was born in Babhnaur (Gaya), Bihar, India, on July 1, 1946. He received the B.Sc. (Eng.) degree from the Bihar Institute of Technology, Sindri, India, and the M.Sc. (Eng.) and Ph.D. degrees from the Birla Institute of Technology (BIT), Ranchi, India, in 1968, 1970, and 1979, respectively.

He has been with the Telecommunications and Traffic Control Systems Group, Delft University of Technology (DUT), The Netherlands, since February 1988. He is Head of the Wireless Transmission Research Section of the International Research Centre for Telecommunications Transmission and Radar (IRCTR), where he is actively involved in the area of personal, indoor, and mobile radio communications. His current research interests are in communications, multiple-access protocols, adaptive equalizers, spread spectrum, CDMA systems, and multimedia communications. $\mathrm{He}$ is the DUT Project Leader for the European Acts project FRAMES and is a Member of the COST-259 project. 